\title{
Epidural steroid injections for lumbar spinal stenosis
}

\author{
Mark A. Harrast
}

Published online: 31 October 2007

(C) Humana Press 2007

\begin{abstract}
Degenerative lumbar stenosis is a common source of pain and disability in the elderly. It presents clinically with a variety of symptoms, though neurogenic claudication is the hallmark. There is a multifactorial pathogenesis to lumbar stenosis and its symptoms, and thus, there are multiple management approaches available. Epidural steroid injections (ESIs) are a popular choice in management, however, the literature is vague in definitive support of their use, and providers that utilize injections can use variable techniques to access the spinal canal in order to deposit the steroid at the appropriate site. This article will review degenerative lumbar stenosis in general and focus on the use of ESIs to better define their role in this management process. In addition, the evidence to discern the optimal injection route will be presented.
\end{abstract}

Keywords Lumbar stenosis - Lumbar stenosis treatment . Epidural steroid injections

\section{Introduction}

Degenerative lumbar spinal stenosis (LSS) is a common source of pain and disability in the elderly population. Neurogenic claudication is the hallmark symptom of LSS, classically described as buttock and bilateral leg pain initiated by walking, prolonged standing, and walking downhill (relative lumbar extension). It is typically relieved by sitting, bending forward, or pushing a grocery cart. This is contrasted with vascular claudication where pain is

M. A. Harrast $(\square)$

Rehabilitation Medicine, Orthopaedics and Sports Medicine, University of Washington, 325 Ninth Avenue, Box 359721, Seattle, WA 98104, USA

e-mail: mharrast@u.washington.edu relieved solely by rest (not having to sit or bend forward), and walking uphill is worse.

Neurogenic claudication typically results from stenosis of the central spinal canal. LSS is a result of the degenerative spine cascade, and thus, narrowing not only can affect the central spinal canal, but also the lateral recesses and intervertebral foramina [1]. Due to the variable regions affected, patients with LSS can present with unilateral or bilateral, and monoradicular or polyradicular symptoms. They can also present with frank radiculopathy, i.e., weakness, sensation loss, and reflex loss in a myotomal and dermatonal distribution. Back pain is also a common complaint of patients with LSS. The quality of this axial symptom is consistent with osteoarthritis of the lumbar spine (stiffness with a dull, aching pain). Patients tend to default to a stooped-forward posture to alleviate pain by widening the spinal canal and decreasing the forces on the zygaphophyseal joints.

The natural history of LSS is not entirely known. However, it is known that rapid neurological progression is rare. Despite methodological limitations, most studies of nonoperative therapy for lumbar stenosis report 15-45\% improvement, 15-30\% worsen, and the rest remain symptomatically about the same [2-5]. In a study with 8-10-year follow-up comparing surgical to nonoperative therapy, early outcomes at 1 and 4 years favored surgical management; however, after 8-10 years, low back pain outcome, predominant symptom (either back or leg pain) improvement, and satisfaction with their current status were similar. Leg pain relief, though, still favored those treated surgically [6].

\section{Multifactorial pathogenesis}

We know that the pathogenesis of LSS is multifactorial. If narrowing and compression were the sole pathologic 
entities of LSS, decompressive surgery would be a panacea. There are vascular, biochemical, and biomechanical factors that contribute to the symptoms of LSS, and thus, need to be considered as much as the physical compression. The vascular factors include venous engorgement and arterial insufficiency of the radicular blood supply, which can lead to an ischemic neuritis.

In the venous engorgement theory, spinal veins dilate during ambulation in stenotic patients, thus, blood flow stagnates and intrathecal pressures rise, which cause a microcirculatory neuroischemic insult and subsequently, claudication symptoms $[7,8]$.

Arterial insufficiency is another proposed source of the claudication symptoms of LSS. With lower limb exercise, including ambulation, the lumbar radicular arterioles dilate to provide nourishment to the spinal nerve roots. In patients with stenosis, however, this arterial dilation may be defective [9].

The inflammatory cascade is another component of this multifactorial pathogenesis. Over the years, multiple inflammatory mediators have been implicated in the pathogenesis of radicular symptoms due to disk herniations, including phospholypase A2, cytokines, nitric oxide, lactate, and immune cells [10]. The antigenic nucleus pulposis leaks out of its immunoprotected environment and induces a local immune response. These inflammatory mediators may enhance the excitability of the dorsal root ganglion under a state of chronic compression from stenosis. However, the true role of immunemediated inflammation has not yet been elucidated in the setting of spinal stenosis. Theoretically, in LSS, mechanical compression of a nerve root may be a "primer" for a subsequent inflammatory response, which ultimately causes the radicular symptoms. This may be an explanation for the patient with chronic LSS to have periodic acute flares of symptoms. A chronically inflamed nerve root, with increased mechanical sensitivity, can become perturbed by a new inflammatory precipitator, vascular changes, or degenerative instability [9].

Patients with LSS have disk and zygapophyseal joint degeneration, which can lead to a degenerative spondylolisthesis. The resultant mechanical instability can cause a "dynamic radiculopathy" by imposing a stretch on the nerve root as it passes through the unstable level. This instability may result in further vascular and inflammatory changes, which contribute to the multifactorial pathogensis of symptoms.

\section{Treatment}

Treatment for LSS includes conservative (activity modification, assistive devices for ambulation, medications,
Table 1 Management of degenerative lumbar stenosis

Conservative

1. Activity modification (limit extension-based activity)

2. Assistive device for ambulation (walker)

3. Medications (Tylenol, NSAIDs, neuromodulating agents, and low dose opiates)

4. Physical therapy and exercise

Interventional

1. Epidural corticosteroid injections

2. Surgery

and exercise) and interventional (ESIs and surgery) approaches. Treatment decisions should be driven in part by patient preference. Some patients would never consider interventional treatments unless all conservative options were exhausted. Others with severe and disabling symptoms may opt for more aggressive management earlier. As clinicians, we need to inform our patients of the possible treatments, and guide them appropriately (See Table 1).

Surgical intervention has variable outcomes, though most studies describe good to excellent overall results $[2,6$, 11]. However, conservative treatment is a viable initial option for many patients. Except in severe cases, there is no evidence that neurologic deterioration occurs over time, and delaying surgery in those choosing initial conservative management does not adversely affect the post-operative outcome [12]. Consider nonoperative management as a means of managing ongoing symptoms and functional deficits as opposed to substantially altering the natural history of LSS.

Epidural steroid injections are frequently used in a nonoperative management regime (Figs. 1 and 2). They should be used as an adjunct to a comprehensive rehabilitation program and not used in isolation. The pain relief obtained with injections can facilitate the patient's tolerance of a rehabilitation program, which is the main component of treatment used to decrease patient disability.

Unfortunately, there is no clear evidence to guide us on when to initiate a trial of epidural steroids, nor to assist in determining the frequency and duration of treatment. Literature does support their use for predominantly radicular symptoms, especially acutely, and less for axial symptoms. The "series of three" is out of fashion now that fluoroscopic guidance and more specific routes of administration are utilized routinely. If one well-placed injection is not effective, then it is unlikely that a second or third administered in the same location will be. However, potentially a different route of administration could be utilized for a second injection. 
There are multiple mechanisms of action of pain relief for corticosteroids. These include the inhibition of nerve root edema with improved microcirculation and reduced ischemia, inhibition of prostaglandin synthesis, and also the noninflammatory action of direct inhibition of C-fiber neuronal membrane excitation [13, 14].

\section{Technique}

There are three injection approaches to access the lumbar epidural space: the interlaminar, caudal, and transforminal techniques. The interlaminar and caudal approaches historically were performed without fluoroscopic guidance; however, there is a $30-40 \%$ miss rate, even in experienced hands, without fluoroscopic guidance, thus, fluoroscopy is recommended for all approaches $[15,16]$.
Interlaminar approach

This procedure can be performanced at any interlaminar level in the lumbar spine, though most commonly is performed at L4-5. The injectant typically stays in the posterior epidural space and can travel a level or two caudad or cephalad, depending on the volume injected. Thus, an L3-4 interlaminar ESI could be considered in the patient with diffuse, nonlocalizing bilateral symptoms of neurogenic claudication with notable L3-4 central stenosis on MRI. However, to access the anterior epidural space, bilateral L4-5 transforaminal approaches may be considered in this same scenario (Fig. 3).

\section{Caudal approach}

The entry point for this approach is the sacral hiatus. Typically, a larger volume of injectant is administered to
Fig. 1 An 64-year-old female with symptoms of bilateral lower limb neurogenic claudication with symptomatic improvement with a caudal epidural steroid injection. An interlaminar approach could have been considered appropriate, as well. (a) Sagittal view of a T2-weighted MRI of the lumbar spine. Note the grade I spondylolisthesis of L4 on L5 with severe central canal stenosis. (b) and (c) Axial views of a T2-weighted MRI through L4-5. Note the diffuse disc bulge in (b) and the marked ligamentum flavum hypertophy in (c), both contributing to the severe central stenosis. (d) The L5-S1 level showing no evidence of stenosis
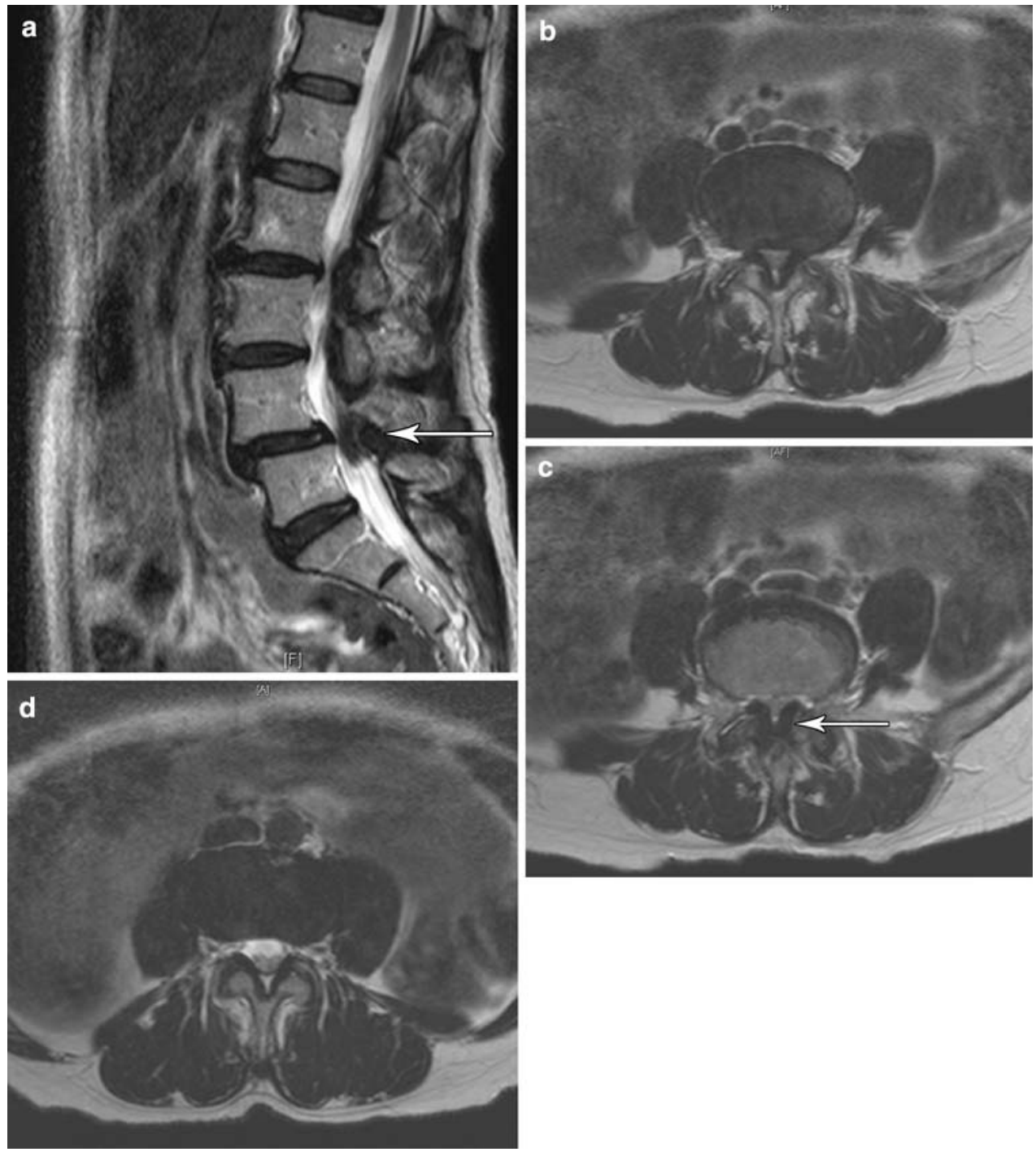
distribute the steroid cephalad in the epidural space. The majority of injectant can reach the L5-S1 and L4-5 levels, thus treating higher level pathology via a caudal approach is not recommended. This approach is used for L4-5 and L5-S1 pathology when symptoms are more diffuse (not monoradicular-when a transforaminal approach would be desirable), or if there is a concern of epidural scarring in a post-operative patient (when performing an interlaminar approach would risk dural puncture). Thus, a caudal ESI could be considered in the patient with nonlocalizing, bilateral symptoms of neurogenic claudication with MRI confirmed L5-S1 central stenosis. Both an interlaminar and caudal route are better for more diffuse symptoms, or when there is multilevel pathology contributing to the patient's symptoms (Fig. 4).

\section{Transforaminal approach}

This approach requires fluoroscopic guidance and is the most selective of the three. The transforaminal approach allows access to the ventral epidural space, where the disk lies, and thus, is thought to be most effective for a monoradiculopathy due to disk herniation. Thus, a patient with right L5 radicular symptoms and MRI confirmed L4-5 central or lateral recess stenosis, may most benefit from a right L5-S1 transforaminal ESI (Fig. 5).

\section{Outcomes}

There are limited studies evaluating the efficacy of ESIs for degenerative lumbar stenosis, as the majority of injection outcome studies are evaluating lumbosacral radiculopathy secondary to disk herniation. There are no studies comparing efficacy of injection techniques in patient with LSS.

Of the four studies evaluating the efficacy of nonfluoroscopically guided lumbar ESIs for LSS, all showed a short-term benefit ranging from 1 week to 2 months of relief, and only one [17] demonstrated a longer term benefit with up to 10 months of relief [1720]. The Ciocon [17] and Hoogmartens [19] studies used a caudal approach, where the Fukusaki [18] and Rosen [20] studies used the interlaminar approach. The more recent studies used fluoroscopic guidance, but also demonstrated variable results [21-24]. All demonstrated some short-term benefit; however, the Botwin study [22], the only prospective evaluation, showed a substantial long-term benefit as well. This study evaluated 34 patients with unilateral radicular symptoms
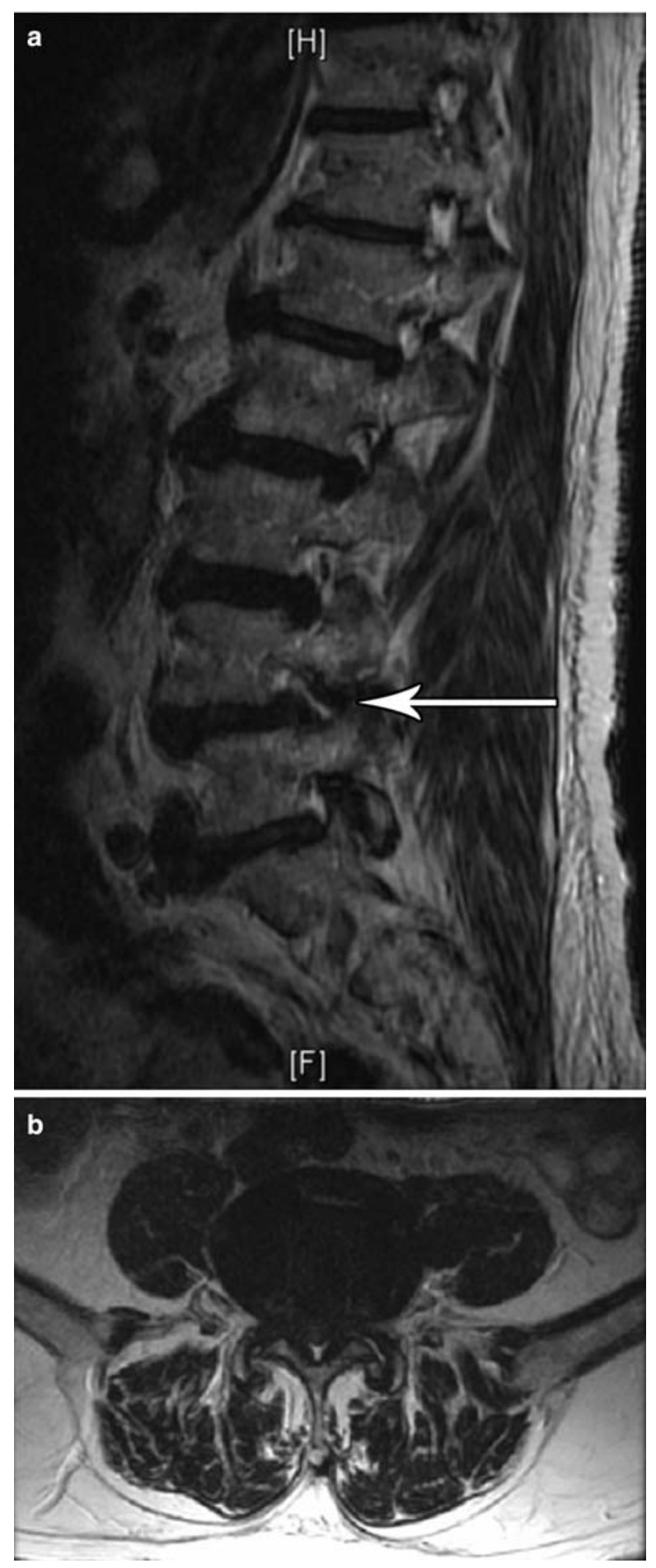

Fig. 2 An 84-year-old male with low back pain and right L4 radicular symptoms to the ankle worsened with walking with symptomatic improvement with a right L4-5 transforaminal epidural steroid injection. (a) Right sagittal view of a T2-weighted MRI of the lumbar spine. Note the multilevel degenerative changes and the foraminal stenosis at L4-5 related to disc bulge and facet hypertrophy. (b) Axial view of a T2-weighted MRI through L4-5. Note severe central stenosis on imaging, though symptomatically, he described right L4 radicular symptoms and thus a transforaminal route was chosen 
Fig. 3 Fluoroscopic images of an L3-4 interlaminar approach. (a) AP view, pre-contrast, (b) Lateral view, pre-contrast, and (c) Lateral view, post-contrast
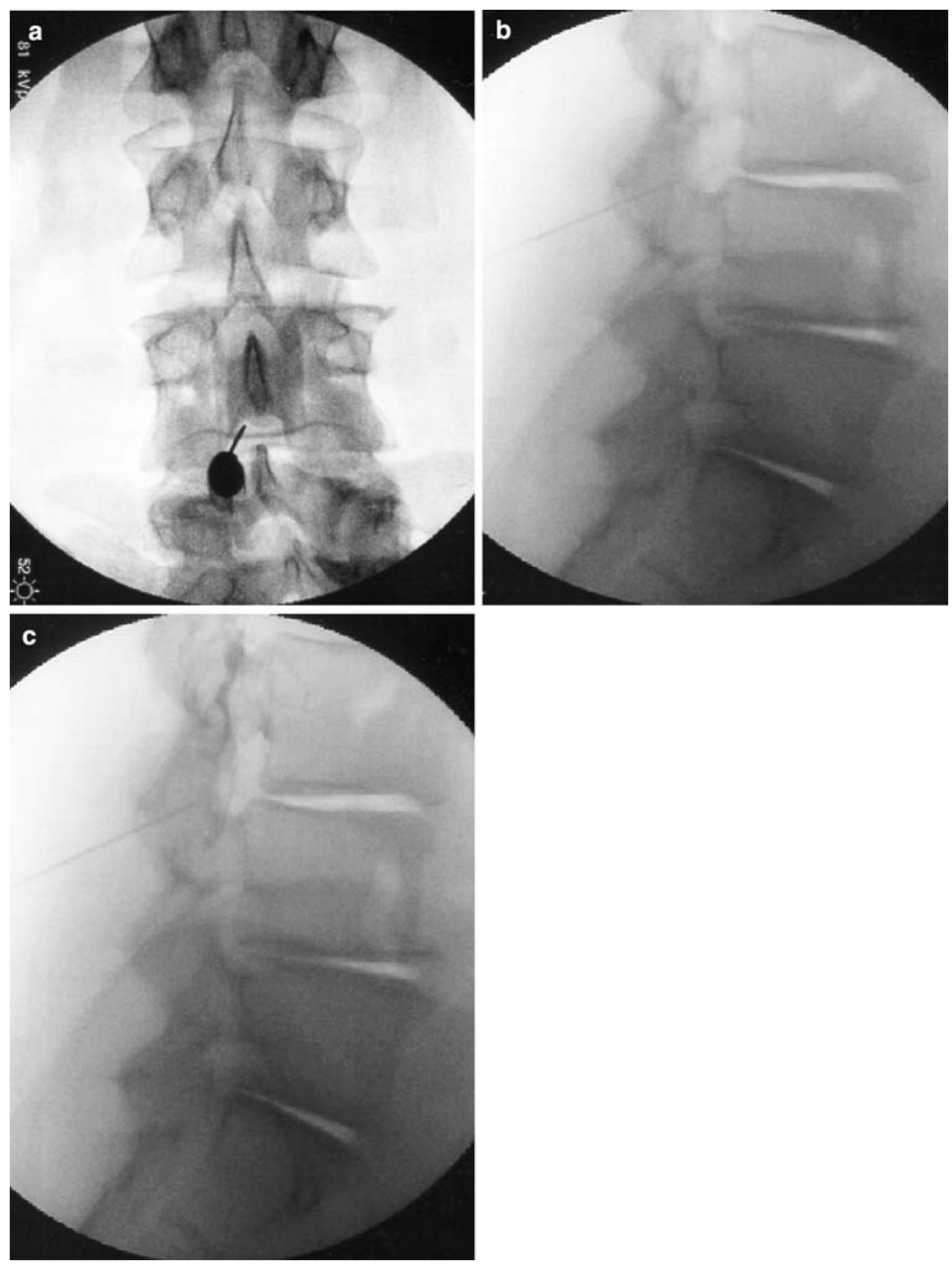

secondary to LSS with fluoroscopically guided tranforaminal ESIs. A mean of 1.9 injections per patient was performed. Subjects were evaluated at 2 months and 1 year with a visual analog scale, Roland 5-point scale, standing/walking tolerance scale, and patient satisfaction scale. All outcome measures demonstrated a statistically significant improvement at 2 and 12 months when compared to the pre-injection baseline. The patient satisfaction scale revealed that $62 \%$ of patients at 2 months and $64 \%$ of patients at 12 months felt somewhat or completely better.

\section{Conclusion}

There is limited research evaluating the appropriate use of lumbar ESIs specifically to treat LSS, and thus, specific conclusions cannot be drawn. There is no information to conclude which injection technique is most efficacious. For now, treatment decisions can be adapted from the literature on injections for symptomatic disc herniation and radiculopathy. These studies confirm that ESIs are best used for acute radicular pain, and that the transforaminal approach can target the primary site of pathology better than an 
Fig. 4 Fluoroscopic images of a caudal approach. (a) Lateral view, pre-contrast and (b) lateral view, post-contrast
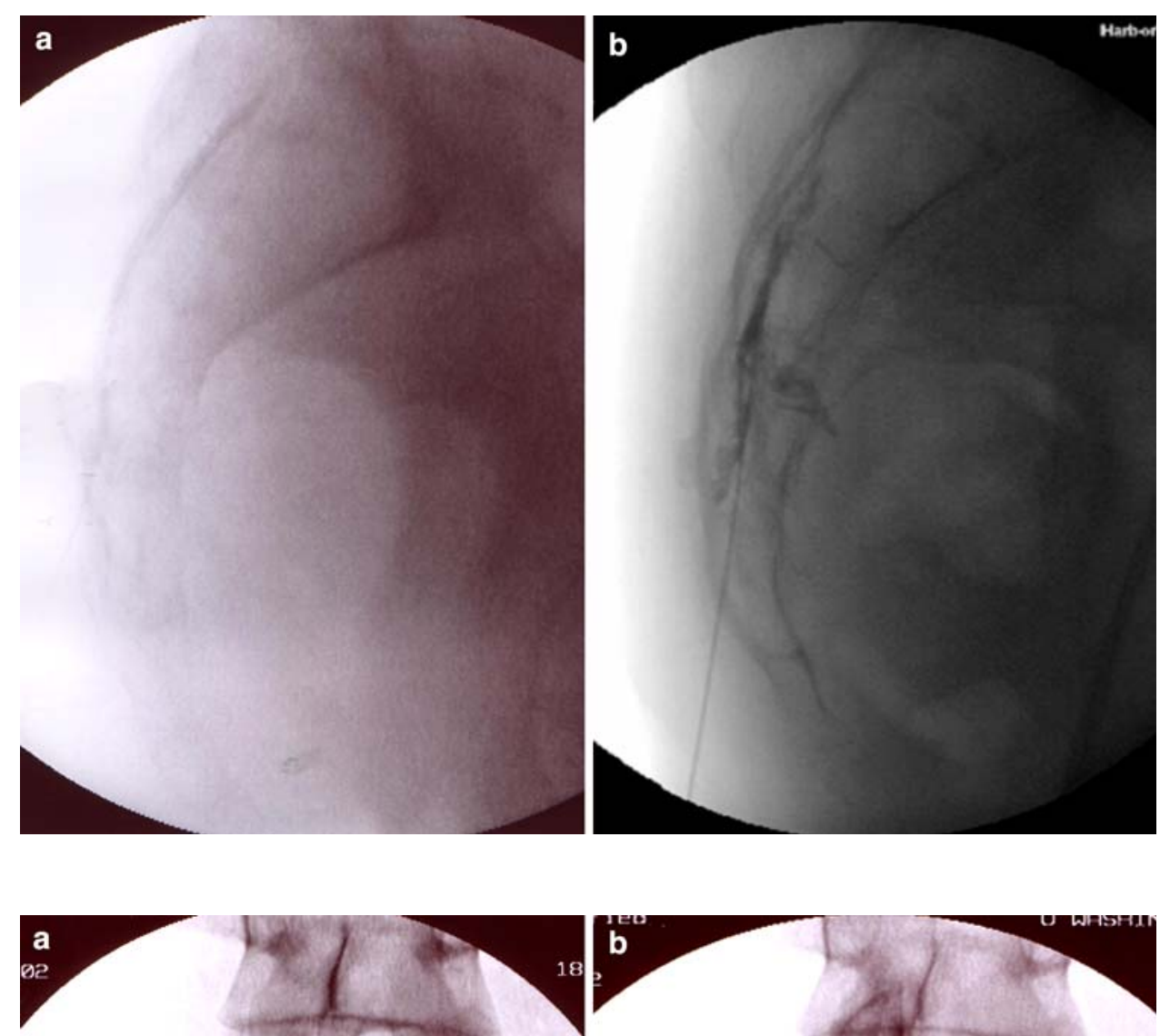
approach targeting the right L5 nerve root. (a) AP view, precontrast and (b) AP view, postcontrast

Fig. 5 Fluoroscopic images of a right L5-S1 transforaminal

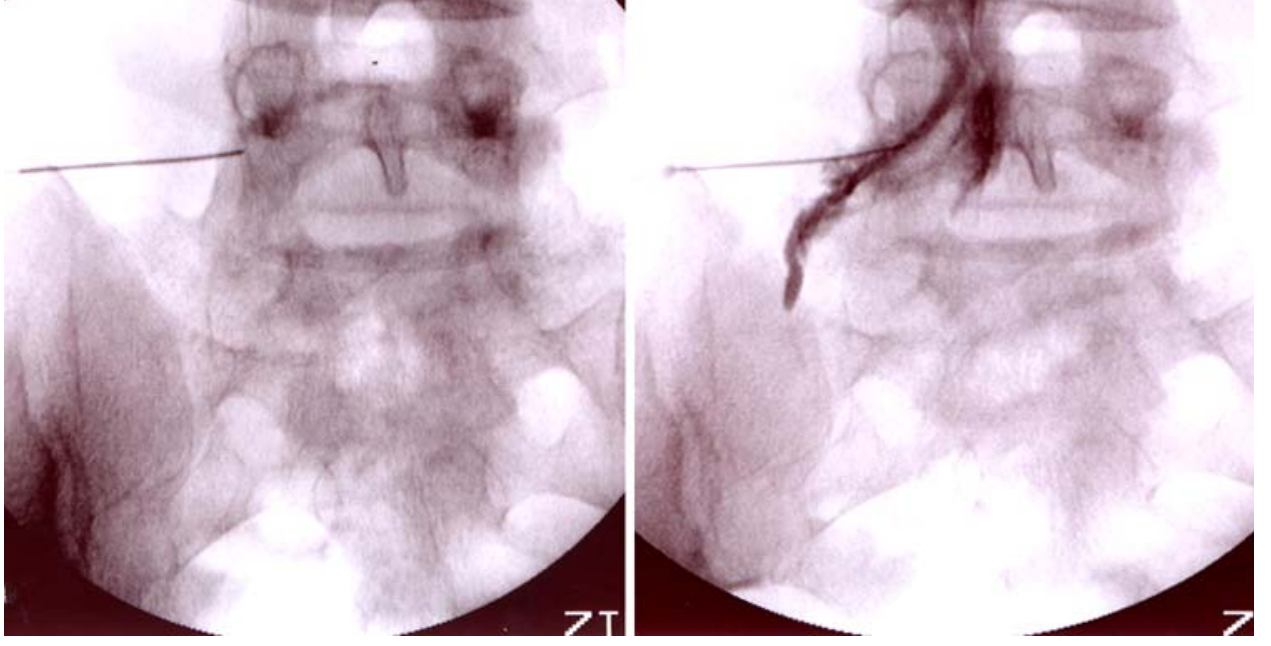

interlaminar approach and thus is probably more efficacious [25-27].

Acknowledgment I would like to express my appreciation to Nilda Gatchalian for assistance in preparing this manuscript.

\section{References}

1. Kirkaldy-Willis WH, Wedge JH, Yong-Hing K, et al. Pathology and pathogenesis of lumbar spondylosis and stenosis. Spine 1978;3:319-28
2. Atlas SJ, Deyo RA, Keller RB, et al. The Maine lumbar spine study, Part III: 1-Year outcomes of surgical and nonsurgical management of lumbar spinal stenosis. Spine 1996;21: 1787-94

3. Johnsson KE, Rosen I, Uden A. The natural course of lumbar spinal stenosis. Clin Orthop 1992;Jun:82-6

4. Simotas AC, Dorey FJ, Hansraj KK, et al. Nonoperative treatment for lumbar spinal stenosis: clinical and outcome Results and a 3-year survivorship analysis. Spine 2000;25:197-204

5. Swezey RL. Outcomes for lumbar stenosis: a 5-year follow-up study. J Cir Rheumatol 1996;2:129-34

6. Atlas SJ, Keller RB, Wu YA, et al. Long-term outcomes of surgical and nonsurgical management of lumbar spinal stenosis: 8 
to 10 year results from the Maine lumbar spine study. Spine 2005;30:936-43

7. Crock HV. The applied anatomy of spinal circulation in spinal stenosis. In: McNeill TW, editor. Lumbar spinal stenosis. St. Louis: Mosby; 1992

8. Ooi Y, MIta F, Satoh Y. Myeloscopic study on lumbar spinal canal stenosis with special reference to intermittent claudication. Spine 1990;15:544-9

9. Akuthota V, Lento P, Sowa G. Pathogenesis of lumbar spinal stenosis pain: why does an asymptomatic stenotic patient flare? Phys Med Rehabil Clin N Am 2003;14:17-28

10. Goupille P, Jayson MI, Valat JP, et al. The role of inflammation in disk herniation-associated radiculopathy. Semin Arthritis Rheum 1998;28:60-7

11. Airaksinen O, Herno A, Turunen V, et al. Surgical outcome of 438 patients treated surgically for lumbar spinal stenosis. Spine 1997;22:2278-82

12. Botwin KP, Gruber RD. Lumbar epidural steroid injections in the patient with lumbar stenosis. Phys Med Rehabil Clin N Am 2003;14:121-41

13. Johansson A, Hao J, Sjolund B. Local corticosteroid application blocks transmission in normal nociceptive C-fibers. Acta Anesthesiol Scand 1990;34:335-58

14. Olmarker K, Byrod G, Cornefjord M, et al. Effects of Methylprednisolone on nucleus pulposus-induced nerve root injury. Spine 1994;19:1803-8

15. Renfrew DL, Morre TE, Kathol MH, et al. Correct placement of epidural steroid injections: fluoroscopic guidance and contrast administration. Am J Neuroradiol 1991;12:1003-7

16. Sitz M, Sommer HM. Accuracy of blind versus fluoroscepically guided caudal epidural injections. Spine 1999;13:1371-6

17. Ciocon JO, Galindo-Ciocon D, Amaranath L, et al. Caudal epidural blocks for elderly patients with lumbar canal stenosis. J Am Geriatric Soc 1994;42:593-6
18. Fukusaki M, Kobayashi I, Hara T, et al. Symptoms of spinal stenosis do not improve after epidural steroid injection. Clin $\mathrm{J}$ Pain 1998;14:148-51

19. Hoogmartens M, Morelle P. Epidural injections in the treatment of spinal stenosis. Acta Orthop Belg 1987;53:409-11

20. Rosen CD, Kahanovitz N, Bernstein R, et al. A retrospective analysis of the efficacy of epidural steroid injections. Clin Orthop $1988 ; 228: 270-2$

21. Barre' L, Lutz GE, Southern D, et al. Fluoroscopically guided caudal epidural steroid injections for lumbar stenosis: a retropective evaluation of long term efficacy. Pain Physician 2004; 7:187-93

22. Botwin KP, Gruber RD, Bouchlas CG, et al. Fluoroscopically guided lumbar transformational epidural steroid injections in degenerative lumbar stenosis: an outcome study. Am J Phys Med Rehabil 2002;81:898-905

23. Cooper G, Lutz GE, Boachie-Adjei O, et al. Effectiveness of transforaminal epidural steroid injections in patients with degenerative lumbar scoliotic stenosis and radiculopathy. Pain Physician 2004;7:311-7

24. Delport EG, Cucuzella AR, Marley JK, et al. Treatment of lumbar spinal stenosis with epidural steroid injections: a retrospective outcome study. Arh Phys Med Rehabil 2004;85:479-84

25. Riew KD, Yin Y, Gilula L, et al. The effect of nerve-root injections on the need for operative treatment of lumbar radicular pain. A prospective, randomized, controlled, double-blind study. J Bone Joint Surg Am 2000;82-A:1589-93

26. Schaufele M, Hatch L, Jones W. Interlaminar versus transforaminal epidural injections for the treatment of symptomatic lumbar intervertebral disc herniations. Pain Physician 2006;9:361-6

27. Vad VB, Bhat AL, Lutz GE, et al. Transforaminal epidural steroid injections in lumbosacral radiculopathy: a prospective randomized study. Spine 2002;27:11-6 